\title{
LHC semileptonic and radiative rare B decays program
}

\author{
P. Koppenburg ${ }^{\mathrm{a}}$, on behalf of the $\mathrm{LHCb}$ collaboration \\ ${ }^{a}$ Imperial College London, Physics Department, London SW7 2BW
}

Rare loop-induced decays are sensitive to New Physics in many Standard Model extensions. In this paper we discuss the reconstruction of the radiative penguin decays $b \rightarrow s \gamma$ and the electroweak penguin decay $b \rightarrow \ell \ell s$ at the LHC. The expected annual yields and $B / S$ estimates are presented.

Studies of the rare radiative penguin decays $\mathrm{b} \rightarrow \mathrm{s} \gamma$, the electroweak penguin decay $\mathrm{b} \rightarrow \ell \ell \mathrm{s}$, and the decay $\mathrm{B}_{\mathrm{s}} \rightarrow \mu \mu[1]$ allow to extract valuable information about penguin and box loop-diagrams. The complex couplings of new particles may result in enhancement of decay rates or in the appearance of non-trivial $\mathcal{C} \mathcal{P}$-violating phases. For example for the decay $B_{d} \rightarrow K^{*} \gamma$ because of the one-diagram dominance (the strong phase appears only at order $\alpha_{\mathrm{S}}$ and $1 / \mathrm{m}_{\mathrm{b}}$ ) the $\operatorname{direct} \mathcal{C P}$ asymmetry is reliably predicted in the SM to be $\leq 1 \%$ [2], but for some SUSY scenarios it could be as large as $10-40 \%[2,3]$.

Due to the $V-A$ structure of the weak current the photon polarisation in $\mathrm{b} \rightarrow \mathrm{s}(\mathrm{d}) \gamma$ transitions is almost $100 \%$. In the SM this causes mixinginduced $\mathcal{C P}$-asymmetries to vanish [4], while in extensions of the SM these asymmetries could be as large as 50\% [5]. This effect can be used as a probe for the spin structure of new particles.

The test of QCD models in radiative penguin decays still plays an important rôle [6]. The ratio $\left|\mathrm{V}_{\mathrm{td}}\right| /\left|\mathrm{V}_{\mathrm{ts}}\right|$ could be extracted from $\Gamma\left(\mathrm{B}_{\mathrm{d}} \rightarrow \omega \gamma\right) /$ $\Gamma\left(\mathrm{B}_{\mathrm{d}} \rightarrow \mathrm{K}^{*} \gamma\right)$ with moderate theoretical uncertainty [7].

The forward-backward asymmetry $A_{\mathrm{FB}}$ for the decay $\mathrm{b} \rightarrow \ell \ell \mathrm{s}$, is defined through the angle $\theta_{\mathrm{FB}}$ between the $\ell^{+}$and the $\mathrm{b}$ hadron flight directions in the di-lepton rest frame. The shape of the asymmetry $A_{\mathrm{FB}}\left(m_{\ell \ell}^{2}\right)$ and especially the position of the zero crossing in the SM are almost unaffected by hadronic form factor uncertainties, thus providing a good basis for searching for deviations [8].

The ratio of $\mathrm{b} \rightarrow \mu \mu \mathrm{s}$ and $\mathrm{b} \rightarrow$ ees decays in any exclusive mode is also a clean probe of the SM. Lepton-universality predicts this ratio to be 1 with theoretical errors below $1 \%$ [9].

The LHC will produce copious amounts of bhadrons, with a total $\mathrm{b} \overline{\mathrm{b}}$ cross-section of $500 \mu \mathrm{b}$. This potential will be exploited by the ATLAS, CMS and $\mathrm{LHCb}$ experiments.

ATLAS and CMS are general-purpose central spectrometers designed for new physics searches at high luminosity [10]. Yet they will have a small trigger bandwidth dedicated to B-physics for decays involving muons during the initial running at lower luminosity. We assume for the following that this programme covers 3 years of running at $\mathcal{L}=10^{33} \mathrm{~cm}^{-2} \mathrm{~s}^{-1}$, i.e. $30 \mathrm{fb}^{-1}$.

$\mathrm{LHCb}$ is a forward spectrometer [11] optimised for $b$ physics. Its main features are the precise vertex detector, the two RICH detectors and the versatile trigger with a $2 \mathrm{kHz}$ output rate dominated by pp $\rightarrow$ b $\overline{b X}$ events. $\mathrm{LHCb}$ will operate at a lower luminosity of $\mathcal{L}=2 \cdot 10^{32} \mathrm{~cm}^{-2} \mathrm{~s}^{-1}$, corresponding to $2 \mathrm{fb}^{-1}$ per year.

The reconstruction of rare $b$ decays at $\mathrm{LHC}$ is a challenge due to the small rates and large backgrounds from various sources. The most critical is the combinatorial background from $\mathrm{pp} \rightarrow \mathrm{b} \overline{\mathrm{b} X}$ events, containing secondary vertices and characterised by high charged and neutral multiplicities.

\section{Radiative $B$ meson decays at $\mathrm{LHCb}$}

Radiative $\mathrm{b} \rightarrow \mathrm{s} \gamma$ decays can be reconstructed in the modes $\mathrm{B}_{\mathrm{d}} \rightarrow \mathrm{K}^{*} \gamma^{1}$ and $\mathrm{B}_{\mathrm{s}} \rightarrow \phi \gamma[12] . \quad \mathrm{K}^{*}$

${ }^{1}$ The charge conjugate mode is always implied unless explicitly stated otherwise 

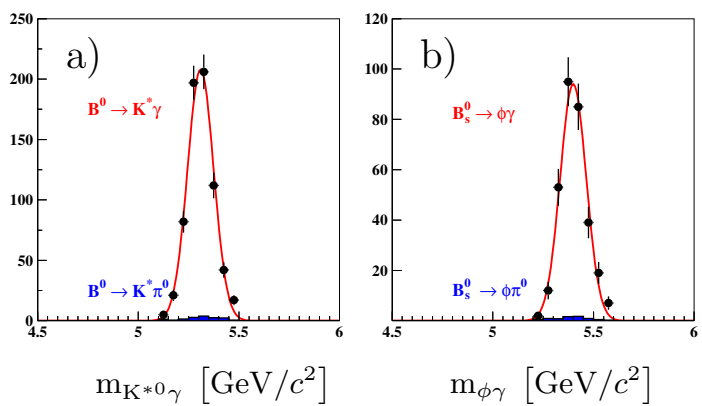

Figure 1. Reconstructed $\mathrm{B}_{\mathrm{d}} \rightarrow \mathrm{K}^{*} \gamma$ and $\mathrm{B}_{\mathrm{s}} \rightarrow \phi \gamma$ mass distributions for signal events after trigger and selection cuts. The specific background from $\mathrm{B} \rightarrow \mathrm{K}^{*} \pi^{0}$ and $\mathrm{B} \rightarrow \phi \pi^{0}$ decays is shown with proper normalisation. The dominant combinatorial background is not shown.

and $\phi$ candidates are reconstructed in the $\mathrm{K}^{+} \pi^{-}$ and $\mathrm{K}^{+} \mathrm{K}^{-}$modes respectively. Charged tracks have to be inconsistent with any reconstructed primary vertex. Selected $\mathrm{K}^{* 0}(\phi)$ candidates are combined with photon candidates of transverse energy greater than $2.8 \mathrm{GeV}$. The reconstructed $\mathrm{B}$ candidate is required to be compatible with coming from a primary vertex. This requirement is one of the most powerful cuts against combinatorial background. Background from the decays $\mathrm{B}_{\mathrm{d}} \rightarrow \mathrm{K}^{*} \pi^{0}$ and $\mathrm{B}_{\mathrm{d}} \rightarrow \phi \pi^{0}$ with an energetic $\pi^{0}$ reconstructed as a single photon is suppressed by cutting on the $\mathrm{K}^{*}$ and $\phi$ helicity angle. The mass resolution of the selected and triggered $\mathrm{B}$ candidates is expected to be $65 \mathrm{MeV} / \mathrm{c}^{2}$ as shown in Figure 1.

The selection of the Cabibbo suppressed decay $\mathrm{B}_{\mathrm{d}} \rightarrow \omega \gamma$ followed by $\omega \rightarrow \pi^{+} \pi^{-} \pi^{0}$ follows a similar approach, but it is complicated by the $\pi^{0}$ reconstruction $[13] .^{2}$

The expected annual $\left(2 \mathrm{fb}^{-1}\right)$ yields and $B / S$ ratios in a $\pm 200 \mathrm{MeV} / c^{2}$ mass window are given in the table below:

\begin{tabular}{lrr} 
& Yield & $B / S$ \\
\hline $\mathrm{B}_{\mathrm{d}} \rightarrow \mathrm{K}^{*} \gamma$ & 35000 & $<0.7$ \\
$\mathrm{~B}_{\mathrm{s}} \rightarrow \phi \gamma$ & 9000 & $<2.4$ \\
\hline $\mathrm{B}_{\mathrm{d}} \rightarrow \omega \gamma$ & 40 & $<3.5$
\end{tabular}

${ }^{2}$ The $\mathrm{B}_{\mathrm{d}} \rightarrow \rho \gamma$ mode has not yet been studied, but is expected to be cleaner than the $\omega$ counterpart.
The background is estimated from a fully simulated $\mathrm{pp} \rightarrow \mathrm{b} \overline{\mathrm{b} X} \mathrm{MC}$ sample. The limits are given at $90 \%$ C.L.

These yields will for instance allow $\mathcal{C P}$ asymmetry measurements at the per-cent level in the $\mathrm{B}_{\mathrm{d}} \rightarrow \mathrm{K}^{*} \gamma$ channel.

\section{2. $\Lambda_{\mathrm{b}} \rightarrow \Lambda \gamma$ at $\mathbf{L H C b}$}

Radiative b baryon decays like $\Lambda_{\mathrm{b}} \rightarrow \Lambda \gamma$ can be used to probe the chirality of the effective Hamiltonian by measuring the photon polarisation [14]. The angular asymmetry between the $\Lambda_{\mathrm{b}}$ spin and the photon momentum combined with the $\Lambda \rightarrow$ $\mathrm{p} \pi$ decay polarisation probes the predicted $V-A$ structure of this decay.

The $\Lambda$ reconstruction is delicate at the $\mathrm{LHC}$ since it may traverse a large fraction of the tracking system before decaying. Therefore one also uses decays to heavier $\Lambda$ resonances decaying strongly to $\mathrm{pK}$, losing the handle from the $\Lambda$ decay polarisation [15].

The event selection is similar to the one presented above for $\mathrm{B}$ mesons. In a preliminary study $\mathrm{LHCb}$ expects the following annual yields and $B / S$ ratios for one year $\left(2 \mathrm{fb}^{-1}\right)$ :

\begin{tabular}{lrc} 
& Yield & $B / S$ \\
\hline$\Lambda_{\mathrm{b}} \rightarrow \Lambda \gamma$ & 750 & $<42$ \\
\hline$\Lambda_{\mathrm{b}} \rightarrow \Lambda(1520) \gamma$ & 4200 & $<10$ \\
$\Lambda_{\mathrm{b}} \rightarrow \Lambda(1670) \gamma$ & 2500 & $<18$ \\
$\Lambda_{\mathrm{b}} \rightarrow \Lambda(1690) \gamma$ & 2500 & $<18$
\end{tabular}

The same comment as in Section 1 applies about the background estimates.

The $\Lambda_{\mathrm{b}}$ is expected to be polarised in pp collisions at LHC, especially in the forward region. This polarisation is assumed to be $20 \%$, a fraction that will be measured to a $1 \%$ precision with $\Lambda_{\mathrm{b}}$ $\rightarrow \mathrm{J} / \psi \Lambda$ decays $[16]$.

With these yields LHCb will be able to measure the right-handed polarisation component to an accuracy of $5 \%$ after 5 years of data taking $\left(10 \mathrm{fb}^{-1}\right)$, which is smaller than the expected SM contribution [17]. This accuracy only depends weakly on the actual value of the $\Lambda_{\mathrm{b}}$ polarisation. 


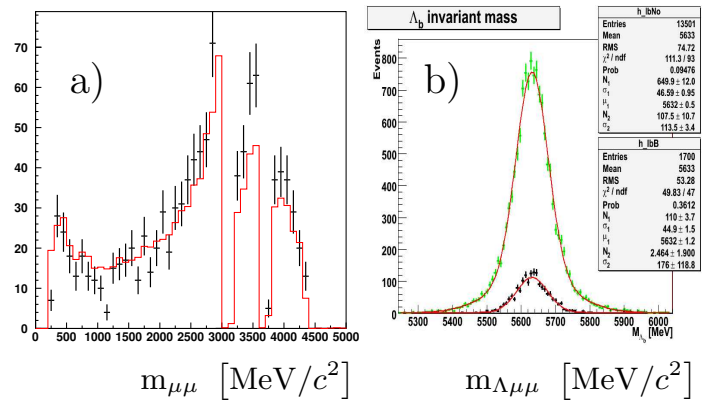

Figure 2. a) The $\mu \mu$ mass in $\mathrm{B}_{\mathrm{d}} \rightarrow \mu \mu \mathrm{K}^{*}$ at LHCb. Selected events (crosses) are compared with generated events (solid). b) The $\Lambda \mu \mu$ mass at ATLAS (all signal and selected event compared).

\section{3. $A_{\mathrm{FB}}$ at the LHC}

Thanks to its very clean experimental signature the decay $\mathrm{b} \rightarrow \mu \mu \mathrm{s}$ can be accessed by ATLAS, CMS and $\mathrm{LHCb}$ in the exclusive decays $\mathrm{B}_{\mathrm{u}} \rightarrow \mu \mu \mathrm{K}, \mathrm{B}_{\mathrm{s}} \rightarrow \mu \mu \phi, \mathrm{B}_{\mathrm{d}} \rightarrow \mu \mu \mathrm{K}^{*}$ and $\Lambda_{\mathrm{b}} \rightarrow \mu \mu \Lambda$. The latter two can be used to extract $A_{\mathrm{FB}}{ }^{3}$

The selections combine two tracks positively identified as opposite-charged muons with the relevant hadronic final state. Similar selection criteria as in Section 1 are applied. Very strict requirements on the vertex quality are applied to reduce the backgrounds from cascade semileptonic $\mathrm{b} \rightarrow$ $\mu \nu \mathrm{c}, \mathrm{c} \rightarrow \mu \nu \mathrm{s}$ decays and from two semileptonic $\mathrm{b} \rightarrow \mu \nu \mathrm{c}$ decays. The former background needs to be well under control because it induces an $A_{\mathrm{FB}}$ bias. The background from c $\overline{\mathrm{c}}$ resonances is removed by vetoing the $\mathrm{J} / \psi$ and $\psi(2 \mathrm{~S})$ mass windows.

$\mathrm{LHCb}$ expects a $15 \mathrm{MeV} / c^{2}$ resolution for the $\mathrm{B}$ mass and $10 \mathrm{MeV} / c^{2}$ for the $\mu \mu$ mass. The resolution for $\theta_{\mathrm{FB}}$ is $4 \mathrm{mrad}$. The distributions for the di-muon invariant mass and the angle $\theta_{\mathrm{FB}}$ are not distorted by acceptance or selection cuts, as illustrated in Fig. 2a for $m_{\mu \mu}$ [18].

ATLAS has also studied these channels and obtains a typical mass resolution of $60 \mathrm{MeV} / c^{2}$ for the final candidates as shown in Fig. $2 \mathrm{~b}$ for the

\footnotetext{
${ }^{3}$ The $A_{\mathrm{FB}}$ in $\mathrm{B}_{\mathrm{u}} \rightarrow \mu \mu \mathrm{K}$ is expected to be null in the $\mathrm{SM}$ and most extensions. $\mathrm{B}_{\mathrm{S}} \rightarrow \mu \mu \phi$ is not self tagging which greatly reduces the sensitivity.
}
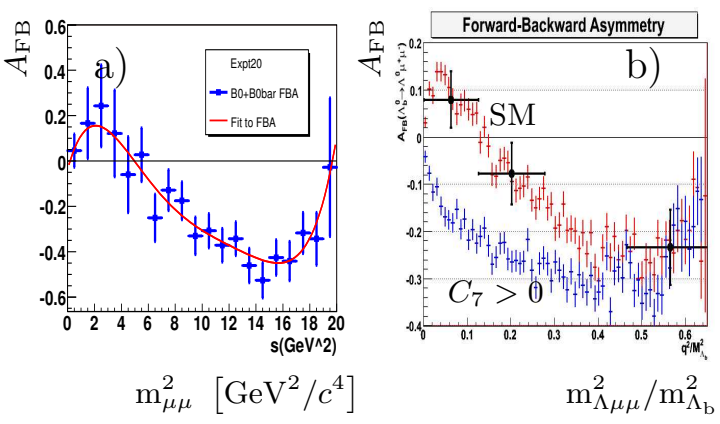

Figure 3. Typical $A_{\mathrm{FB}}$ versus di-lepton mass plots for a) $\mathrm{B}_{\mathrm{d}} \rightarrow \mu \mu \mathrm{K}^{*}$ with $2 \mathrm{fb}^{-1}$ at $\mathrm{LHCb}$ and b) $\Lambda_{\mathrm{b}} \rightarrow \mu \mu \Lambda$ with $30 \mathrm{fb}^{-1}$ at ATLAS compared to two theoretical expectations.

decay $\Lambda_{\mathrm{b}} \rightarrow \mu \mu \Lambda$.

The expected yields for these channels are listed below for one nominal year of running at $\mathrm{LHCb}$ and three years of running at ATLAS.

\begin{tabular}{lclrr} 
& $\int \mathcal{L} \mathrm{d} t$ & & Yield & $B / S$ \\
\hline LHCb & $2 \mathrm{fb}^{-1}$ & $\mathrm{~B}_{\mathrm{d}} \rightarrow \mu \mu \mathrm{K}^{*}$ & 4400 & $<3$ \\
LHCb & $2 \mathrm{fb}^{-1}$ & $\mathrm{~B}_{\mathrm{u}} \rightarrow \mu \mu \mathrm{K}$ & 1600 & $\sim 3$ \\
\hline ATLAS & $30 \mathrm{fb}^{-1}$ & $\mathrm{~B}_{\mathrm{d}} \rightarrow \mu \mu \mathrm{K}^{*}$ & 2500 & $<20$ \\
ATLAS & $30 \mathrm{fb}^{-1}$ & $\mathrm{~B}_{\mathrm{u}} \rightarrow \mu \mu \mathrm{K}$ & 1500 & $<6$ \\
ATLAS & $30 \mathrm{fb}^{-1}$ & $\mathrm{~B}_{\mathrm{s}} \rightarrow \mu \mu \phi$ & 900 & $<11$ \\
ATLAS & $30 \mathrm{fb}^{-1}$ & $\Lambda_{\mathrm{b}} \rightarrow \mu \mu \Lambda$ & 800 & $<5$
\end{tabular}

LHCb estimates its sensitivity to $A_{\mathrm{FB}}$ in $\mathrm{B}_{\mathrm{d}} \rightarrow \mu \mu \mathrm{K}^{*}$ in a toy MC study using these yields, $B / S$ and the relevant distributions. A typical year of running could provide the $A_{\mathrm{FB}}$ versus $m_{\mu \mu}^{2}$ plot shown in Fig. 3a, already allowing to exhibit non-SM features. $\mathrm{LHCb}$ expects to extract the $C_{9} / C_{7}$ Wilson-coefficients ratio from the crossing point with the $A_{\mathrm{FB}}=0$ axis to a precision of $13 \%$ after 5 years of running $\left(10 \mathrm{fb}^{-1}\right)$.

ATLAS will also be able to disentangle the SM expectation from extensions with $C_{7}>0$ after three years $\left(30 \mathrm{fb}^{-1}\right)$, as shown in Fig. $3 \mathrm{~b}$ for $\Lambda_{\mathrm{b}} \rightarrow \mu \mu \Lambda$.

\section{4. $R_{\mathrm{K}}$ at $\mathrm{LHCb}$}

Reconstructing $\mathrm{B}_{\mathrm{u}} \rightarrow \mathrm{eeK}$ as well as $\mathrm{B}_{\mathrm{u}} \rightarrow \mu \mu \mathrm{K}$ allows us to extract the ratio $R_{\mathrm{K}}$ of the two branching fractions, integrated over a given di- 


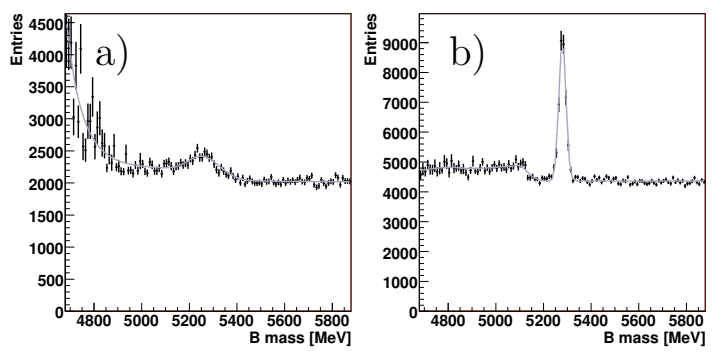

Figure 4. Expected B candidate mass distributions in a) $\mathrm{B}_{\mathrm{u}} \rightarrow$ eeK and b) $\mathrm{B}_{\mathrm{u}} \rightarrow \mu \mu \mathrm{K}$ modes for $10 \mathrm{fb}^{-1}$ at $\mathrm{LHCb}$.

lepton mass range. ${ }^{4} \quad \mathrm{~B}_{\mathrm{u}} \rightarrow \mu \mu \mathrm{K}$ decays are reconstructed as explained above and the same requirements are applied for the $\mathrm{B}_{\mathrm{u}} \rightarrow$ eeK decay. A proper bremsstrahlung correction is essential in this channel. The correction for the lower reconstruction and trigger efficiency in the electron mode is extracted from $\mathrm{B}_{\mathrm{u}} \rightarrow \mathrm{J} / \psi \mathrm{K}$ decays. The di-lepton mass range is chosen to be $1<m_{\ell \ell}^{2}<6 \mathrm{GeV}^{2} / \mathrm{c}^{4}$ in order to avoid c $\overline{\mathrm{c}}$ resonances (especially in the ee mode) and thresholds effect due to the higher $\mu$ mass. The event yields are extracted from a two-dimensional fit to the $\ell \ell \mathrm{K}$ and $\ell \ell$ masses in order to take into account the backgrounds from $\mathrm{b} \rightarrow \mathrm{J} / \psi \mathrm{s}$ and $\mathrm{B} \rightarrow \ell \ell \mathrm{K}^{*}$.

The expected $\mathrm{B}$ candidate mass distributions are shown in Fig. 4 for five years $\left(10 \mathrm{fb}^{-1}\right)$ of data taking. The yields are:

\begin{tabular}{lcrc} 
& Yield & $B / S$ & $\sigma\left(m_{\mathrm{B}}\right)$ \\
\hline $\mathrm{B}_{\mathrm{u}} \rightarrow \mu \mu \mathrm{K}$ & $8000 \pm 50$ & $\sim 3$ & $15 \mathrm{MeV} / \mathrm{c}^{2}$ \\
$\mathrm{~B}_{\mathrm{u}} \rightarrow \mathrm{eeK}$ & $1800 \pm 35$ & $\sim 5$ & $75 \mathrm{MeV} / \mathrm{c}^{2}$
\end{tabular}

The errors on the yields are the statistical error in the estimate. Using these errors one gets an error on $R_{\mathrm{K}}$ of $4 \%$ after five years of running $\left(10 \mathrm{fb}^{-1}\right)$.

\section{Conclusion}

The LHC experiments have a promising physics potential for the study of numerous loop-induced rare decays such as the radiative penguin decays $\mathrm{b} \rightarrow \mathrm{s} \gamma$ and the electroweak penguin decay $\mathrm{b} \rightarrow \ell \ell \mathrm{s}$.

${ }^{4} \mathrm{~B}_{\mathrm{d}} \rightarrow \ell \ell \mathrm{K}^{*}$ is also a good candidate (measuring $R_{\mathrm{K}^{*}}$ ) but has not yet been studied.
The expected annual signal event yields and preliminary estimates on background-to-signal ratios have been presented.

The precision and reliability of background-tosignal estimates are expected to improve with a significant increase of Monte Carlo samples. Studies of high level trigger efficiencies, systematic uncertainties, and the sensitivity to new physics are in progress.

The author would like to thank P. Řezníček, M. Smižanská, I. Belyaev and U. Egede for useful discussions.

\section{REFERENCES}

1. P. Řezníček, ibid.

2. T. Hurth and T. Mannel, AIP Conf. Proc.602:212-219 (2001).

3. M. Neubert, Supersymmetry and Unification Conf. 2002, hep-ph/0212360.

4. M. Gronau, Rencontres de Moriond 2003, hep-ph/0304240.

5. D. Atwood, M. Gronau and A. Soni, Phys. Rev. Lett. 79185 (1997).

6. M. Neubert, Int. J. Mod. Phys. A17:29362950,2002 (2001).

7. D. Mohapatra et al., the Belle Collaboration, Phys. Rev. Lett. 96 (2006) 221601.

8. A. Ali, ICHEP 2002, hep-ph/0210183.

9. G. Hiller, F. Krüger, Phys. Rev. D69:074020 (2004).

10. T. Speer, ibid. and references therein.

11. F. Metlica, ibid. and references therein.

12. G. Pakhlova and I. Belyaev, LHCb-2003-090.

13. O. Deschamps et al., LHCb-2003-091.

14. T. Mannel, S. Recksiegel, Nucl. Part. Phys 24 (1998) 979-990.

15. F. Legger, T. Schietinger, hep-ph/0605245. F. Legger, Ph.D. Thesis, EPFL, 2006.

16. J. Hřivnáč, R. Lednický, M. Smižanská, hepph/9405231.

17. B. Grinstein et al., Phys. Rev. D71 (2005) 011504.

18. J.H. Lopes, LHCb-2003-104. 